\title{
Anti-CD30/CD16A Monoclonal Antibody AFM13
}

National Cancer Institute

\section{Source}

National Cancer Institute. Anti-CD30/CD16A Monoclonal Antibody AFM13. NCI

Thesaurus. Code C94219.

A tetravalent bispecific antibody directed against human CD30 and the human low affinity IgG Fc region receptor (FCGR3A; CD16A), with potential immunomodulating and antineoplastic activities. Anti-CD30/CD16A monoclonal antibody AFM13 binds to the CD16A expressed on natural killer (NK) cells with two of its binding sites and to CD30 on CD30-expressing tumor cells with the other two binding sites, thereby selectively crosslinking tumor and NK cells. This may result in NK cell activation, antibody-dependent cellular cytotoxicity (ADCC) and eventually tumor cell lysis. CD30, a cell surface receptor and a member of the tumor necrosis factor (TNF) receptor superfamily, is overexpressed in hematologic malignancies; CD16A is specifically expressed on the surface of NK cells. 